\title{
A novel transcription factor PunR and Nac are involved in purine and purine nucleoside transporter punC regulation in E. coli
}

Irina Rodionova ( $\sim$ irodionova@ucsd.edu )

UCSD https://orcid.org/0000-0002-6500-2758

\section{Ye Gao}

University of California at San Diego

Anand Sastry

University of California at San Diego

\section{Ying Hefner}

University of California at San Diego

\section{Reo Yoo}

University of California at San Diego

\section{Dmitry Rodionov}

Sanford-Burnham-Prebys Medical Discovery Institute

\section{Milton Saier}

University of California at San Diego

\section{Bernhard Palsson}

University of California, San Diego https://orcid.org/0000-0003-2357-6785

\section{Article}

Keywords: punR, punC, E. coli, Nac

Posted Date: February 19th, 2021

DOl: https://doi.org/10.21203/rs.3.rs-146218/v1

License: (c) (i) This work is licensed under a Creative Commons Attribution 4.0 International License.

Read Full License

Version of Record: A version of this preprint was published at Communications Biology on August 19th, 2021. See the published version at https://doi.org/10.1038/s42003-021-02516-0. 


\section{Abstract}

Many genes in bacterial genomes are of unknown function, often referred to as y-genes. Recently, novel analytic methods have divided bacterial transcriptomes into independently modulated sets of genes (iModulons). Functionally annotated iModulons that contain y-genes lead to testable hypotheses to elucidate y-gene function. Inversely correlated expression of a putative transporter gene, ydhC, relative to purine biosynthetic genes, has led to the hypothesis that it encodes a purine-related transporter and revealed a LysR-family regulator, YdhB, with a predicted 23-bp palindromic binding motif. RNA-Seq analysis of a ydhB knockout mutant confirmed the YdhB-dependent activation of ydhC in the presence of adenosine. The deletion of either the ydhC or the ydhB gene led to a substantially decreased growth rate for E. coli in minimal medium with adenosine as the nitrogen source, as well as with inosine or guanosine. Taken together, we provide clear evidence that YdhB activates the expression of the ydhC gene that encodes a novel purine transporter in E. coli. We propose that the genes ydhB and ydhC be renamed as punR and punC, respectively.

\section{Introduction}

At least one third of the genes in the growing number of available sequenced microbial genomes are still of unknown function. Furthermore, the functional annotations of a large portion of the remaining genes are based exclusively on sequence similarity to a relatively small pool of experimentally studied proteins. Even the best-studied model bacterium, Escherichia coli K-12 MG165, is not an exception, despite the long-term effort to curate available knowledge bases. EcoCyc is the premier example of a curated knowledge-base that summarizes functional evidence for over 4,500 genes, including 1567 enzymes, 282 transporters, and 204 transcription factors (1). Previous efforts to construct a global functional atlas of $E$. coli proteins revealed that 1,431 of $4,225(35 \%)$ protein coding genes were not functionally annotated as of 2019 (2, 3) according to three criteria: (i) the gene name starts with ' $y$ ' (hence, it is a y-gene); (ii) it does not have a linked pathway in EcoCyc; and (iii) it has a 'predicted', 'hypothetical', or 'conserved' protein functional description in GenProtEC (4). The COMBREX knowledge base further reveals that experimental evidence is lacking for $44 \%$ of E. coliproteins (5).

The bioinformatic prediction and experimental confirmation of microbial gene functions is a challenging problem. Bioinformatic analyses for the prediction of gene regulation, gene clustering analysis, and metabolic pathway reconstruction lead to reliable hypotheses, followed by experimental verification (68). Such discovery efforts for specific classes of gene functions, such as transcription factors (TFs), is bearing fruit $(9,10)$, leading to a comprehensive list of 278 TFs in E. coli.

The transcriptional regulation of purine/pyrimidine biosynthesis and the salvage regulon are coordinated by the transcriptional regulator PurR in Enterobacteria. We previously identified groups of genes, each of which independently but co-ordinately modulated under different conditions, called iModulons (11). iModulons differ from regulons in that they are computed as independent source signals in a set of transcriptomic data sets (a dynamic measure). In contrast, regulons are defined by TF binding sites in the 
promoters of the regulated genes (a static measure). iModulons showed considerable overlap with previously characterized regulons (11). In some cases, iModulons split such regulons into two dynamically regulated sets of genes, thus, creating two iModulons corresponding to a single regulon.

One example highlighted in this study is the PurR-1 iModulon, which contains genes related to purine biosynthesis and utilization, as a part of the PurR regulon (Fig. 1) (11). Genes encoding the purine biosynthetic pathway (pur) and the transporters ( $g h x P, x a n P)$ were found to be repressed in the presence of exogenous adenine, whereas two genes were upregulated in the presence of adenine: add, encoding adenosine deaminase and involved in purine catabolism/salvage, and $y d h C$, encoding a putative transporter of unknown function from the Drug: $\mathrm{H}^{+}$Antiporter-1 (DHA1) family (TCDB family ID 2.A.1.2). The expression of these genes was inversely correlated with purine biosynthesis gene expression (11). In particular, the add gene is directly activated by the ribose-responsive transcriptional regulator RbsR, while the transcription of purHD is repressed by RbsR (12), suggesting the dependency of purine biosynthesis on intracellular ribose concentration.

The purine and pyrimidine de novo biosynthetic group of genes is directly controlled by PurR; however, the regulatory mechanism for $y d h C$ was not reported. YdhC had previously been detected as an arabinose efflux transporter in rich medium (13), suggesting a relationship to pentose efflux and possibly the nutrient uptake from the rich medium.

Here, we identify a LysR-family regulator and propose that YdhB directly controls the transcription of YdhC in response to adenine. We also provide evidence that $\mathrm{YdhC}$ is a purine and purine nucleoside transporter (Fig. 1) and that YdhC is important for the resistance to sulfonamides. Machine learningbased analysis of RNA-Seq data was a useful tool for studying the co-expression of genes and identified the $y d h B C$ genes as targets for investigation. Transcriptional activation of $y d h C$ by the nitrogen assimilation control regulator Nac was shown by RNA-Seq analysis of the nac mutant in the adenine supplemented medium. The work described here expands our understanding of how purine uptake for salvage and degradation versus biosynthesis is reciprocally regulated in $E$. coli.

\section{Results}

Systems analysis and the prediction of PunC function. The reconstruction of purine salvage and utilization under different conditions shows that adenosine/adenine and guanosine/guanine transporters are important as mediators of the uptake of purines when present in the medium as a supplement or substitute for a more traditional carbon or nitrogen source. Guanosine, inosine, cytidine, and thymidine (but not uridine, adenosine, and xanthosine) are transported by NupG (14). The pyrimidine nucleoside: $\mathrm{H}^{+}$ symporter, NupC, has been characterized $(14,15)$. NupG and NupC can recognize the nucleoside ribose moiety (14) and are regulated by the global carbon catabolite repressor protein, CRP, as well as pyrimidine sensor/regulators CytR and DeoR (16). The NupC and NupG transporters are activated by the presence of cytidine. But no difference for nupG expression noticed in the presence of adenine as supplement to $\mathrm{M} 9$ medium (ModulonDB). NupC and NupG were shown recently to be important ADP-glucose uptake 
transporters, and they are essential for the incorporation of extracellular ADP-glucose into glycogen during biosynthesis (16). The coordinate regulation of different purine/pyrimidine transporters is important for the utilization of nucleosides as carbon and nitrogen sources or nucleoside salvage under various starvation conditions (17).

Adenosine as a nitrogen source is utilized via adenosine deaminase (Add), which converts it to inosine (Fig. 1). PpnP is a broad specificity pyrimidine/purine nucleoside phosphorylase that produces hypoxanthine (a purine base) and D-ribose-1-phoshate from phosphate and inosine (the corresponding nucleoside), respectively (18). However, it also acts on uridine, adenosine, guanosine, cytidine, thymidine, and xanthosine as substrates.

The PurR regulon is essential for purine biosynthesis when salvage is limited. PurR repression is activated by the presence of hypoxanthine or guanine, signalling that the purine cytoplasmic concentration is sufficient for growth $(17,19,20)$. The inverse correlation for the modulation of mRNA levels for PurR-regulated purine biosynthetic genes and those of the $y d h C$ and add genes (21) has been demonstrated during growth in minimal medium with glucose and adenine as a supplement, suggesting a role in purine salvage (11). For PurR regulon related genes, the levels of mRNAs encoding proteins of purine biosynthesis and guanosine uptake (PurR-1 iModulon) decrease in the presence of adenine in contrast to the high increase of $y d h C$ mRNA level $(11,19)$, but no changes in mRNA level was found for nupC and nupG.

Under the conditions described here, extracellular adenosine or adenine is the only nitrogen source supporting the growth of $E$. coli, but it does not support growth of a $y d h C$ deletion mutant strain. The hypoxanthine produced from adenosine is a signal for the PurR-mediated repression of purine biosynthesis. This proposed regulation of the PurR-1 iModulon and reconstruction of purine biosynthesis and purine uptake is shown in Fig. 1. The reconstruction of purine biosynthesis and regulation is important for the gene function hypothesis. Independent component analysis revealed the transcriptional regulation of the Pur-1 iModulon, consisting of the purB, purC, purD, purE, purF, purH, purK, purl, purM, purN, purT genes and those encoding the corresponding transporters, as well as the inverse regulation of the transcription of the $y d h C$ and add genes in response to the presence of adenine in the growth medium (11). Thus, purine biosynthesis pathway genes and those encoding the guanine/hypoxanthine and xanthine transporters $(g h x P, x a n P)$, are repressed by PurR in response to the availability of cellular hypoxanthine (Fig. 1). Extracellular adenosine/adenine potentially gives rise to hypoxanthine due to uptake followed by the action of adenosine deaminase, and further, by the PpnP-catalyzed reaction (Fig. 1), but the adenosine uptake transporter for nitrogen source utilization was not found.

We hypothesize that $\mathrm{YdhC}$ is an adenosine/adenine transporter, re-named PunC, because the presence of a purine source in the medium inhibits biosynthesis (via the PurR-dependent repression), while upregulating PunC. Although NupG is a known purine transporter, regulation by CRP and CytR suggests that NupG is essential for uptake when purine is to be used as a carbon source. NupG requires hydroxyl groups in the ribose moiety for substrate binding and is not utilized for adenine uptake. The punC 
(b1660) regulation by nitrogen assimilation protein (Nac) was detected by RNA-Seq according to published data (22) (ModulonDB). Nac activates the pathways for utilization of histidine, proline, urea, and alanine in Klebsiella pneumoniae, a related enterobacteria (23). The physiological function of PunC is likely to supplement $E$. coli growth with purine/purine nucleobase to be used as a nitrogen source or during purine salvage.

Prediction of PunR binding sites. The previously uncharacterized transcriptional regulator YdhB (renamed PunR here) belongs to the LysR family of bacterial transcription factors. The PunR gene is located in a conserved gene cluster with the divergently transcribed punC gene, and this arrangement is conserved in other Proteobacteria (Fig. S1). The punR promoter is predicted to be Sigma 24-dependent, and the punC promoter is Sigma 24- and Sigma 70-dependent (RegulonDB). To identify and characterize DNA binding sites of PunR in the E. coli genome, we utilized combined bioinformatic and experimental approaches. We applied a comparative genomic approach of phylogenetic footprinting (24) to predict the putative PunR-binding site in the common intergenic region between the punR and punC genes (Fig. S2). Alignment of the upstream regions for the punC genes from three groups of Enterobacteria revealed conserved motifs with a common 23-bp palindromic consensus for binding of the predicted PunR regulator (Fig. 2). A similar palindromic motif was also identified in the punC/ punR intergenic region in Pseudomonas spp. (Fig. 2), confirming strong conservation of the predicted PunR binding site across gamma-proteobacteria. A common motif of these orthologous operators is an imperfect palindrome with consensus TsttwTCAAwAwwwTTGaaGGCA, where ' $s$ ' is either $\mathrm{G}$ or $\mathrm{C}$ and ' $w$ ' is either A or T.

Experimental validation of the PunR binding site. We conducted ChIP-exo studies in M9 glucose medium supplemented with $2.5 \mathrm{mM}$ adenosine to validate the predicted PunR operator and searched for additional candidate PunR binding sites in the E. coligenome (Fig. 2). The chromosomal punR gene was genetically modified to encode a Myc-tagged PunR. The E. coli strain was grown with glucose as the carbon source in M9 minimal media, and the ChIP-exo experiment was designed to pull down PunR which occupied an $\sim 200$ bp region upstream of punC. The PunR-protected region from E. colicorresponds to the PunR binding site predicted by phylogenetic footprinting (Fig. 2).

We further conducted fluorescent polarization (FP) assays to confirm PunR binding to its predicted 23-bp DNA motif. The purified PunR_His protein (40\% purity) or refolded protein was incubated in the assay mixture with a fluorescently labelled DNA fragment (as described in Materials and Methods), predicted by phylogenetic footprinting. PunR $(0-60 \mathrm{nM})$ was incubated with a 29-bp DNA fragment containing the predicted PunR-binding site plus a $10 \mathrm{mM} \mathrm{MgSO}_{4}$ supplement for 1 hour at room temperature in the presence and the absence of adenine. The FP signals at different concentrations of PunR are shown in Fig. 3, but the binding for the refolded PunR had not been detected. PunR binding in the presence, but not the absence, of $0.35 \mathrm{mM}$ adenine was measured using the fluorescence polarization assay. The PunR binding constant $K_{d}=27 \mathrm{nM}$, for binding to the DNA, was calculated using Prism 7 .

RNA-Seq analysis of the PunR regulon in adenosine or adenine supplemented media. RNA-Seq analysis of the punR mutant of E. coli MG1655 (WT) in M9 with glucose as the carbon source (to exclude NupG 
overproduction), supplemented with $2.5 \mathrm{mM}$ adenosine, showed downregulation of the punC gene. The punR deletion strain of E. coli MG1655 showed a lower expression level of punC (Table 1), while the level of other mRNAs was not changed, suggesting that PunR is a specific activator for punC expression. The location of the PunR binding site upstream of the predicted punC promoter agrees with the PunRdependent activation of punC (Fig. S2). Supplementation of M9 medium with adenosine increased punC mRNA levels only in the presence, but not in the absence, of PunR.

Table 1

RNA-Seq measured differentially expressed gene punC in punR deletion mutant strains compared to $E$. coli MG1655 wild type strain.

\begin{tabular}{|lllllr|}
\hline $\begin{array}{l}\text { Gene } \\
\text { name }\end{array}$ & $\begin{array}{l}\text { Locus } \\
\text { tag }\end{array}$ & $\begin{array}{l}\text { Gene function } \\
\text { (Uniprot) }\end{array}$ & Mean & $\begin{array}{l}\log _{2} \text { Fold } \\
\text { Change }\end{array}$ & P-value \\
\hline punC & b1660 & $\begin{array}{l}\text { Inner membrane transport } \\
\text { protein }\end{array}$ & 33.9 & -2.55 & $1.05 \mathrm{e}-6$ \\
\hline
\end{tabular}

Independent component analysis allows the comparison of the iModulon activities for growth in M9 medium and growth with adenosine supplementation as the sole nitrogen source. The iModulon involved in motility and flagellar synthesis and regulated by the minor sigma factor FliA was downregulated in the WT strain (Fig. S3), but not in the punR mutant strain. FliA regulates the motility and biofilm formation in E. coli and is regulated by FlhDC (11). The flhDC operon is subject to regulation at the transcriptional level by more transcriptional regulatory proteins than any other known operon in E. coli (25).

The upregulation of punC has been shown by RNA-Seq analysis according to published data (22) for the E. coli MG1655 (WT) in M9 minimal medium in the presence, but not in the absence of adenine (iModulonDB) and revealed that punC regulation is Nac-dependent (additionally to PunR). The punC expression profile analysis revealed upregulation during growth in $\mathrm{M} 9$ medium supplemented with $10 \mathrm{mM}$ adenine in the presence of Nac, but not in the absence (Fig. 4A), suggesting that both regulators are essential for the punC transcriptional activation. The volcano plot for the differentially expressed genes compared to the nac mutant is shown (Fig. 4B).

Growth in M9 medium with adenosine or 2-deoxyadenosine as the nitrogen source and with glucose or glycerol as the carbon source. Two E. coli strains, MG1655 and BW25113, have been tested with glucose as the carbon source, when adenosine was added to the M9 medium. The difference between two strains has been shown by the iModulons analysis (11). Under nitrogen limiting conditions, growth of $E$. coli BW25113 was stimulated, but no growth enhancement was observed for the punR or punC mutants (Fig. 5A), but no difference in $\mathrm{M} 9$ medium with $\mathrm{NH}_{4} \mathrm{Cl}$ was detected (Fig. 5B). The punR mutant of $E$. coli MG1655 has a similar growth phenotype under the same conditions (Fig. 5C). When the nupG mutant was tested with the same M9 medium ( $2.5 \mathrm{mM}$ adenosine as the nitrogen source) no growth phenotype was observed (data not shown). 
When the punC and add mutants of E. coli BW25113 were examined with $0.4 \%$ glycerol as the carbon source and $2.5 \mathrm{mM}$ adenosine as the nitrogen source, the growth effect was substantial (the growth phenotypes are shown in Fig. 6A). The growth phenotype with $5 \mathrm{mM}$ 2-deoxyadenosine for the punC mutant was determined, while the add deletion had no effect on growth. These effects are shown in Fig. 6D. We suggest that high concentrations of adenosine/2-deoxyadenosine can support metabolism via the pentose phosphate pathway under nitrogen/carbon starvation, and that PunC is the major transporter under these conditions.

Growth in M9 medium with guanosine (or inosine) and glutamate as nitrogen sources and glycerol as the carbon source. Supplementation of M9 minimal medium with glycerol as the sole carbon source and glutamate as the nitrogen source did not support growth of E. coli. The effect of either inosine or guanosine as supplement as the nitrogen sources was examined using the WT and isogenic add and punC deletion mutant strains, measuring growth when glycerol was the carbon source. The punC mutant showed a decrease in the growth rate compared to the WT strain under nitrogen starvation conditions when inosine or guanosine was present as an additional nitrogen source (Fig. 6B and C). This suggested that the PunC transporter can take up, in addition to adenosine and 2-deoxyadenosine, inosine and guanosine. Thus, the PunC uptake transporter exhibits broad specificity for purine nucleosides as has been revealed by these phenotypic analyses under different conditions.

PunC specificity screening for carbon sources using Biolog plate 1 with $\mathrm{NH}_{4} \mathrm{Cl}$ as the nitrogen source. The growth observed for wild type E. coli BW25113 and its isogenic punC mutant (WT) using different carbon sources, including adenosine or inosine, on Biolog plate 1 was measured using M9 minimal medium without another carbon source. The strains were grown overnight in LB and washed twice with M9 medium without the addition of a carbon source. The cultures were diluted in M9 medium without a carbon source, and $0.1 \mathrm{ml}$ of each was added to the 96-well plates. Growth was detected with Omnilog, but no difference for adenosine or other carbon sources was detected for the mutant and WT strain under microaerobic conditions. The growth of the punC deletion mutant in minimal medium with adenosine as the carbon source was likely supported by NupG transporter.

PunC transporter specificity screening for antibiotic sensitivities using Biolog plates $11 \mathrm{C}$ and 12 . We screened for the possible PunC-mediated transport of various antibiotics using the punC mutant compared to the E. coli BW25113 strain. For these purposes, we used Biolog plates 11C and 12. Each of these plates contained four different concentrations of 24 different antibiotics. The punC mutant and WT strains showed the same resistance for all of the included antibiotics except sulfonamides: sulfathiazole, sulfadiazine and sulfamethoxazole. The punC mutant effect has been shown at different sulfonamides concentration (Fig. 7). The increased resistance observed for the punC deletion mutant strain suggests that it exhibits specificity for sulfonamides. The sulfonamide group of antibiotics are structural analogs of para-aminobenzoic acid (PABA). Mercaptoguanine derivatives inhibit conversion of PABA and 6hydroxy-methyldihydropterin-PP to dihydropteroate (26). It is interesting that a distant punC paralogous gene in E. coli encodes Bcr - a bicyclomycin/sulfonamide resistance protein (27). Bcr has been shown to be involved in the export of L-cysteine (28). In contrast, PunC appears to be an adenine/2- 
deoxyadenosine/adenosine/guanosine/inosine uptake transporter with broad purine specificity, that may allow uptake of sulfamethoxazole and sulfothiazole, but not sulfamethazine. The analysis of $E$. coli inhibition by sulfonamides structural analogs shows the PunC specificity for different sulfonamides modifications (Fig. 7).

\section{Discussion}

A new RNA-Seq data analytic approach was applied to study the PurR regulon and PurR-1 iModulon (see iModulonDB.org). The inverse relationship in the PurR-1 i-Modulon, between the expression of purine biosynthetic genes and punC, led to the hypothesis that punC encodes a purine transporter. We addressed this hypothesis and showed that: 1) expression of the putative transporter, PunC, is essential for adenosine, inosine, guanosine, and 2-deoxyadenosine uptake/growth under nitrogen (nitrogen/carbon) starvation conditions, but not for growth using adenosine as a carbon source in M9 medium; 2) the upregulation of the punC gene in the presence of adenine in $\mathrm{M} 9$ medium is the nitrogen assimilation regulatory protein, Nac, dependent. The analysis of RNA-Seq data for the nac mutant during growth in the medium supplemented with $10 \mathrm{mM}$ adenine showed the activation of punC only in the presence of naC; PunC is additionally regulated by the LysR family $\mathrm{HTH}$-type transcriptional regulator PunR, and the punR gene is conserved within the punC genome context. The punC transporter is upregulated under nitrogen starvation conditions, consistent with the sigma-24 (sigma-E) dependent promoter catalogued RegulonDB (29); and 3) The PunC dependent uptake of sulfathiazole and sulfamethoxazole was implied from the fact that the punC mutant strain has substantially increased resistance under microaerobic conditions compared to wild type E. coli BW25113.

The $E$. coli punC deletion mutant has a growth defect in a minimal medium with either 2-deoxyadenosine, adenosine, inosine, or guanosine as the nitrogen source. The predicted DNA-binding for PunR to the palindromic sequence between punR and punC, conserved in many Proteobacteria, serves as the PunR binding site. PunR binding was demonstrated for E. coli using ChIP-exo and a fluorescent polarization assay. We suggest that PunR functions as an activating transcriptional regulator. This hypothesis is supported by the RNA-Seq analysis of the punR mutant and the fact that PunR binding has been detected in the presence, but not in the absence, of adenine by the FP method. The punC gene is present in many Proteobacterial genomes, including all Enterobacteria. The same is true for the punR and purR genes, which are conserved in the same gene contexts (Fig. S1). It is interesting that PunR had previously been shown to be essential for Yersinia pseudotuberculosis growth (30), suggesting that adenosine uptake may be a property of many Proteobacteria. The Yersinia pseudotuberculosis NupG transporter ortholog is absent, but the NupC ortholog is present.

PunR is also essential for growth during nitrogen starvation with purine as the sole nitrogen source and glycerol as the carbon source (Fig. 5). It is interesting that PunR had previously been shown to be essential for Yersinia pseudotuberculosis growth (30), and the NupG ortholog is absent in this bacteria, suggesting that PunC dependent adenosine/guanosine uptake may be a property of many 
Proteobacteria. A punC mutant was found to be more resistant to representative toxic nucleobase analogs, suggesting an uptake function.

The presence of adenosine as the sole nitrogen source can support $E$. coli growth, and it may be involved in acid resistance because the deletion of add attenuates growth in the presence of adenosine under acidic conditions (31). The hypothesis that PunC is involved in acid resistance in the presence of adenosine has been proposed, but under aerobic growth conditions at pH 5.5 with adenosine or inosine as a supplement, PunC dependent growth phenotypes have not been detected in the BW25113 strain (data not shown).

Adenine, adenosine, and deoxyadenosine can be converted to the guanine nucleotide via the salvage pathway and support growth in minimal medium (32). The presence of inosine or guanosine in the $E$. coli growth minimal medium increase the growth rate with a poor nitrogen source as with glutamate and glycerol (sole carbon source), and PunC is essential for the growth. The poor fitness phenotype for an punC mutant, encoding a homologous transporter in Pseudomonas simiae WCS417 (66\% identity with the E.coli protein), has been demonstrated during growth in minimal medium with glucose and adenine as the nitrogen source (33), but mildly important with adenosine as the nitrogen source (fit.genomics.lbl.gov), then adenine transport function for the Pseudomonas simiae PunC is suggested. The PunC broad specificity function in Klebsiella michiganensis M5al is supported by the strong fitness phenotype for the punC mutant homolog (75\% identity with the $E$. coli homolog). This mutant was found to have a strong negative fitness during growth with any one of several purines and purine nucleosides as carbon sources: inosine, 2-deoxyinosine, 2-deoxyadenosine, and 2-deoxyadenosine 5-phosphate, as well as a mild negative effect with adenosine as the carbon source. All of these observations substantiate the main conclusion of this paper that PunC is an adenosine/inosine transporter in E. coli, but the PunC homologous transporter in Klebsiella michiganensis likely has broad specificity for 2-deoxy-inosine/2deoxyadenosine/2-deoxyadenosine-phosphate.

Taken together, the data analysis approach used here shows that PunC family representatives are regulated by PunR and Nac. The transcriptional analysis revealed that the putative transporter encoded by punC is upregulated by $\mathrm{Nac}$ and is correlated with PurR regulation. It is interesting that Nac also upregulates L-histidine biosynthesis genes and pili synthesis in M9 supplemented with adenine (Fig. 4). The experimental verification of the novel PunC transporter function revealed that PunC has a broad specificity to purines and important under nitrogen limited conditions. punC is transcriptionally regulated by the LysR-family regulator PunR and Nac. Nac previously had been shown as adaptor to NtrC protein, probably PunR-Nac complex is essential for the activation. PunR is the transcriptional activator for the punC gene in the presence of adenine, confirmed by experimental verification using RNA-Seq, fluorescent polarisation, and ChIP-exo. The screening of the data represented in the iModulonDB, RegulonDB, EcoCyc, Fitness Browser, and PubSEED databases/platforms will produce new hypothesis for $y$-gene functions.

\section{Materials And Methods}


Bacterial strains and growth conditions. The punR mutant of E. coli MG1655 strain was constructed as described in (9). The punR, add and punC mutant strains of E. coli BW25113 from the Keio single-gene knockout collection (34) or punR mutant of E. coli MG1655 and wild type were grown overnight in LB medium. The punR, add and punC gene deletions were confirmed by PCR using gene specific primers. The cells were refreshed for 3 hours, washed with M9 salts medium and inoculated in the M9 medium with $0.4 \%$ glycerol or $0.2 \%$ glucose as the carbon source and lacking the usual a nitrogen source, but substituting it with $2.5 \mathrm{mM}$ adenosine, $5 \mathrm{mM}$ 2-deoxyadenosine or $\mathrm{M} 9$ supplemented by $5 \mathrm{mM}$ of Lglutamate and $5 \mathrm{mM}$ inosine or guanosine as nitrogen sources. The $E$. coli BW25113 wild type and punC mutant were grown overnight in LB medium, washed by M9 medium and inoculated as recommended to the Omnolog plates $11 \mathrm{C}$ and $12 \mathrm{~B}$ for the antibiotic resistance measurements.

Overproduction of PunR protein. The punR overexpressing strain of E. coli was inoculated from the ASKA collection (35) onto LB agar plates containing chloramphenicol. Overnight cultures were then inoculated from single colonies. Each of the new cultures $(50 \mathrm{ml})$ was started, and after the $\mathrm{OD}_{600}$ reached 0.8 , $0.8 \mathrm{mM}$ IPTG was added. The cultures were incubated at $24^{\circ} \mathrm{C}$ overnight with continuous shaking, and cells were collected by centrifugation. The PunR recombinant protein, containing an $\mathrm{N}$-terminal $6 \mathrm{His}$ tag, was purified by Ni-chelation chromatography from the soluble fraction as described $(36,37)$. The insoluble fraction was solubilized in $7 \mathrm{M}$ urea and purified on a Ni-NTA minicolumn with At-buffer (50 mM Tris- $\mathrm{HCl}$ buffer, $\mathrm{pH} 8,0.5 \mathrm{mM} \mathrm{NaCl}, 5 \mathrm{mM}$ imidazole, and $0.3 \%$ Brij) with $7 \mathrm{M}$ urea. The PunR refolding was effected on the column in the At-buffer. The purification procedure has been described in detail (36).

Identification of putative PunR binding sites by comparative genomics. The potential PunR-binding sites were identified by a phylogenetic footprinting approach using multiple sequence alignments (Fig. S2). Orthologs of the $E$. coli punC and punR genes in other proteobacteria, as well as multiple sequence alignments of orthologous upstream regions, were identified using the PubSEED comparative genomics platform (38). PunR-binding site sequence logos were constructed using the WebLogo tool (39).

RNA sequencing. RNA sequencing data were generated after growth under aerobic exponential growth conditions in M9 medium supplemented with adenosine. The wild type MG1655 strain was grown as a control for the isogenic punR mutant strain. Pre-cultures for the RNA sequencing experiments were started for the growing the cells in LB medium. Cells were then washed twice with M9 medium and inoculated at an $\mathrm{OD}_{600}$ of 0.05 . The cells were collected at an $\mathrm{OD}_{600}$ of 0.6 and were harvested using the Qiagen RNA-protect bacterial reagent according to the manufacturer's specifications. Pelleted cells were stored at $-80^{\circ} \mathrm{C}$, and after cell resuspension and partial lysis, they were ruptured with a bead beater; the total RNA was extracted using a Qiagen RNA purification kit. After total RNA extraction, the quality was assessed using an Aglient Bioanalyser using an RNA 6000 kit after removal of ribosomal RNA. Paired-end strand specific RNA sequencing libraries were prepared as described (9).

Raw-sequencing reads were collected from GEO and mapped to the reference genome (NC_000913.3) using bowtie (v1.1.2) with the following options "-X 1000 -n 2-3 3". Transcript abundance was quantified using summarizeOverlaps from the R GenomicAlignments package (v1.18.0) with the following options: 
"mode = "IntersectionStrict", singleEnd = FALSE, ignore.strand = FALSE, preprocess .reads = invertStrand". To ensure the quality of the compendium, genes shorter than 100 nucleotides and genes with under 10 fragments per million-mapped reads across all samples were removed before further analysis.

Transcripts per million (TPM) were calculated by DESeq2 (v1.22.1). The final expression compendium was log-transformed $\log _{2}(T P M+1)$ before analysis, erred to as log-TPM. Biological replicates with $R^{2}<$ 0.9 between log-TPM were removed to reduce technical noise.

ChIP-exo experiments. The strains harboring 8-myc were generated by a $\lambda$ red-mediated site-specific recombination system, targeting the $\mathrm{C}$-terminal region as described previously (40). ChIP-exo experimentation was performed following the procedures previously described $(10,41)$. To identify PunR binding sites for each strain, the DNA bound to PunR from formaldehyde cross-linked cells, collected after growth in M9 supplemented with adenosine, was isolated by chromatin immunoprecipitation (ChIP) with the antibodies that specifically recognize the myc tag (9E10, Santa Cruz Biotechnology), and Dynabeads Pan Mouse IgG magnetic beads (Invitrogen) were added, followed by stringent washings as described previously (9). ChIP materials (chromatin-beads) were used to perform on-bead enzymatic reactions of the ChIP-exo method. Briefly, the sheared DNA of the chromatin-beads was repaired by the NEBNext End Repair Module (New England Biolabs), followed by the addition of a single dA overhang and ligation of the first adaptor (5'-phosphorylated) using a dA-Tailing Module (New England Biolabs) and NEBNext Quick Ligation Module (New England Biolabs), respectively. Nick repair was performed by using the PreCR Repair Mix (New England Biolabs). Lambda exonuclease- and RecJf exonuclease-treated chromatin was eluted from the beads, and overnight incubation at 65 degrees reversed the protein-DNA cross-link. RNA- and protein-free DNA samples were used to perform primer extension and second adaptor ligation with the following modifications. The DNA samples, incubated for primer extension as described previously (9), were treated with the dA-Tailing Module and NEBNext Quick Ligation Module (New England Biolabs) for second adaptor ligation. The DNA sample, purified using the GeneRead Size Selection Kit (Qiagen), was enriched by polymerase chain reaction (PCR) using Phusion High-Fidelity DNA Polymerase (New England Biolabs). The amplified DNA samples were purified again with a GeneRead Size Selection Kit (Qiagen) and quantified using Qubit dsDNA HS Assay Kit (Life Technologies). The quality of the DNA sample was checked by running the Agilent High Sensitivity DNA Kit using an Agilent 2100 Bioanalyzer before sequencing using HiSeq 2500 (Illumina) following the manufacturer's instructions. Each modified step was also performed following the manufacturer's instructions. ChIP-exo experiments were performed in duplicate.

PunR fluorescent polarization assay. The recombinant PunR protein with $\mathrm{N}$-terminal $\mathrm{His}_{6}$ tag was overproduced in an E. coli strain from the ASKA collection (35). The PunR-producing strain was grown overnight, inoculated into a $50 \mathrm{ml}$ culture, and induced with $0.6 \mathrm{mM} \mathrm{IPTG}$ after an $\mathrm{OD}_{600}=0.6$ was reached. The cells were harvested after 4 hours and lysed as previously described (42). The purified protein and a $10 \mathrm{nM}$ fluorescently labelled DNA fragment (5'-AGG GGG-3') were incubated in the assay mixture. The PunR binding assay mixture $(0.1 \mathrm{ml})$ contained Tris buffer, $\mathrm{pH} 7.5,0.1 \mathrm{M} \mathrm{NaCl}, 0.5 \mathrm{mM}$ EDTA, $10 \mathrm{mM} \mathrm{MgSO}{ }_{4}, 2 \mathrm{mM}$ DTT, $5 \mu \mathrm{g} / \mathrm{ml}$ sperm DNA and $1 \mu \mathrm{M}$ of the fluorescently labelled predicted 
PunR binding DNA fragment as well as 0-0.6 mM adenine. Then the PunR protein $(0-1 \mu \mathrm{M})$ was added to the assay mixture, and it was incubated for 1 hour at $30^{\circ} \mathrm{C}$.

\section{Declarations}

\section{Conflict of interest}

The authors declare that they have no conflict of interest with respect to the contents of this article.

\section{Acknowledgements}

This work was supported by National Institutes of Health (NIH) grants U01Al124316 and GM077402, and Novo Nordisk Foundation Grant Number NNF10CC1016517. We would like to thank A. Bobkova and M. Abrams (UCSD) for editing the manuscript.

\section{References}

1. Keseler IM, Mackie A, Santos-Zavaleta A, Billington R, Bonavides-Martinez C, Caspi R, Fulcher C, Gama-Castro S, Kothari A, Krummenacker M, Latendresse M, Muniz-Rascado L, Ong Q, Paley S, Peralta-Gil M, Subhraveti P, Velazquez-Ramirez DA, Weaver D, Collado-Vides J, Paulsen I, Karp PD. 2017. The EcoCyc database: reflecting new knowledge about Escherichia coli K-12. Nucleic Acids Res 45:D543-D550.

2. Hu P, Janga SC, Babu M, Diaz-Mejia JJ, Butland G, Yang W, Pogoutse O, Guo X, Phanse S, Wong P, Chandran S, Christopoulos C, Nazarians-Armavil A, Nasseri NK, Musso G, Ali M, Nazemof N, Eroukova V, Golshani A, Paccanaro A, Greenblatt JF, Moreno-Hagelsieb G, Emili A. 2009. Global functional atlas of Escherichia coli encompassing previously uncharacterized proteins. PLoS Biol 7:e96.

3. Ghatak S, King ZA, Sastry A, Palsson BO. 2019. The y-ome defines the $35 \%$ of Escherichia coli genes that lack experimental evidence of function. Nucleic Acids Res 47:2446-2454.

4. Serres MH, Goswami S, Riley M. 2004. GenProtEC: an updated and improved analysis of functions of Escherichia coli K-12 proteins. Nucleic Acids Res 32:D300-2.

5. Anton BP, Chang YC, Brown P, Choi HP, Faller LL, Guleria J, Hu Z, Klitgord N, Levy-Moonshine A, Maksad A, Mazumdar V, McGettrick M, Osmani L, Pokrzywa R, Rachlin J, Swaminathan R, Allen B, Housman G, Monahan C, Rochussen K, Tao K, Bhagwat AS, Brenner SE, Columbus L, de CrecyLagard V, Ferguson D, Fomenkov A, Gadda G, Morgan RD, Osterman AL, Rodionov DA, Rodionova IA, Rudd KE, Soll D, Spain J, Xu SY, Bateman A, Blumenthal RM, Bollinger JM, Chang WS, Ferrer M, Friedberg I, Galperin MY, Gobeill J, Haft D, Hunt J, Karp P, Klimke W, Krebs C, Macelis D, et al. 2013. The COMBREX project: design, methodology, and initial results. PLoS Biol 11:e1001638. 
6. Rodionova IA, Li X, Thiel V, Stolyar S, Stanton K, Fredrickson JK, Bryant DA, Osterman AL, Best AA, Rodionov DA. 2013. Comparative genomics and functional analysis of rhamnose catabolic pathways and regulons in bacteria. Front Microbiol 4:407.

7. Rodionova IA, Scott DA, Grishin NV, Osterman AL, Rodionov DA. 2012. Tagaturonate-fructuronate epimerase UxaE, a novel enzyme in the hexuronate catabolic network in Thermotoga maritima. Environ Microbiol 14:2920-34.

8. Rodionova IA, Vetting MW, Li X, Almo SC, Osterman AL, Rodionov DA. 2017. A novel bifunctional transcriptional regulator of riboflavin metabolism in Archaea. Nucleic Acids Res 45:3785-3799.

9. Gao Y, Yurkovich JT, Seo SW, Kabimoldayev I, Drager A, Chen K, Sastry AV, Fang X, Mih N, Yang L, Eichner J, Cho BK, Kim D, Palsson BO. 2018. Systematic discovery of uncharacterized transcription factors in Escherichia coli K-12 MG1655. Nucleic Acids Res 46:10682-10696.

10. Kim D, Seo SW, Gao Y, Nam H, Guzman GI, Cho BK, Palsson BO. 2018. Systems assessment of transcriptional regulation on central carbon metabolism by Cra and CRP. Nucleic Acids Res 46:29012917.

11. Sastry AV, Gao Y, Szubin R, Hefner Y, Xu S, Kim D, Choudhary KS, Yang L, King ZA, Palsson BO. 2019. The Escherichia coli transcriptome mostly consists of independently regulated modules. Nat Commun 10:5536.

12. Shimada T, Kori A, Ishihama A. 2013. Involvement of the ribose operon repressor RbsR in regulation of purine nucleotide synthesis in Escherichia coli. FEMS Microbiol Lett 344:159-65.

13. Koita K, Rao CV. 2012. Identification and analysis of the putative pentose sugar efflux transporters in Escherichia coli. PLoS One 7:e43700.

14. Patching SG, Baldwin SA, Baldwin AD, Young JD, Gallagher MP, Henderson PJ, Herbert RB. 2005. The nucleoside transport proteins, NupC and NupG, from Escherichia coli: specific structural motifs necessary for the binding of ligands. Org Biomol Chem 3:462-70.

15. Craig JE, Zhang Y, Gallagher MP. 1994. Cloning of the nupC gene of Escherichia coli encoding a nucleoside transport system, and identification of an adjacent insertion element, IS 186. Mol Microbiol 11:1159-68.

16. Almagro G, Viale AM, Montero M, Munoz FJ, Baroja-Fernandez E, Mori H, Pozueta-Romero J. 2018. A CAMP/CRP-controlled mechanism for the incorporation of extracellular ADP-glucose in Escherichia coli involving NupC and NupG nucleoside transporters. Sci Rep 8:15509.

17. Wu H, Li Y, Ma Q, Li Q, Jia Z, Yang B, Xu Q, Fan X, Zhang C, Chen N, Xie X. 2018. Metabolic engineering of Escherichia coli for high-yield uridine production. Metab Eng 49:248-256.

18. Sevin DC, Fuhrer T, Zamboni N, Sauer U. 2017. Nontargeted in vitro metabolomics for highthroughput identification of novel enzymes in Escherichia coli. Nat Methods 14:187-194.

19. Cho BK, Federowicz SA, Embree M, Park YS, Kim D, Palsson BO. 2011. The PurR regulon in Escherichia coli K-12 MG1655. Nucleic Acids Res 39:6456-64.

20. Meng LM, Nygaard P. 1990. Identification of hypoxanthine and guanine as the co-repressors for the purine regulon genes of Escherichia coli. Mol Microbiol 4:2187-92. 
21. Chang ZY, Nygaard P, Chinault AC, Kellems RE. 1991. Deduced amino acid sequence of Escherichia coli adenosine deaminase reveals evolutionarily conserved amino acid residues: implications for catalytic function. Biochemistry 30:2273-80.

22. Bordbar A, Nagarajan H, Lewis NE, Latif H, Ebrahim A, Federowicz S, Schellenberger J, Palsson BO. 2014. Minimal metabolic pathway structure is consistent with associated biomolecular interactions. Mol Syst Biol 10:737.

23. Bender RA. 2010. A NAC for regulating metabolism: the nitrogen assimilation control protein (NAC) from Klebsiella pneumoniae. J Bacteriol 192:4801-11.

24. Rodionov DA. 2007. Comparative genomic reconstruction of transcriptional regulatory networks in bacteria. Chem Rev 107:3467-97.

25. Zhang Z, Kukita C, Humayun MZ, Saier MH. 2017. Environment-directed activation of the Escherichia coliflhDC operon by transposons. Microbiology (Reading) 163:554-569.

26. Dennis ML, Lee MD, Harjani JR, Ahmed M, DeBono AJ, Pitcher NP, Wang ZC, Chhabra S, Barlow N, Rahmani R, Cleary B, Dolezal O, Hattarki M, Aurelio L, Shonberg J, Graham B, Peat TS, Baell JB, Swarbrick JD. 2018. 8-Mercaptoguanine Derivatives as Inhibitors of Dihydropteroate Synthase. Chemistry 24:1922-1930.

27. Nishino K, Yamaguchi A. 2001. Analysis of a complete library of putative drug transporter genes in Escherichia coli. J Bacteriol 183:5803-12.

28. Yamada S, Awano N, Inubushi K, Maeda E, Nakamori S, Nishino K, Yamaguchi A, Takagi H. 2006. Effect of drug transporter genes on cysteine export and overproduction in Escherichia coli. Appl Environ Microbiol 72:4735-42.

29. Santos-Zavaleta A, Salgado H, Gama-Castro S, Sanchez-Perez M, Gomez-Romero L, Ledezma-Tejeida D, Garcia-Sotelo JS, Alquicira-Hernandez K, Muniz-Rascado LJ, Pena-Loredo P, Ishida-Gutierrez C, Velazquez-Ramirez DA, Del Moral-Chavez V, Bonavides-Martinez C, Mendez-Cruz CF, Galagan J, Collado-Vides J. 2019. RegulonDB v 10.5: tackling challenges to unify classic and high throughput knowledge of gene regulation in E. coli K-12. Nucleic Acids Res 47:D212-D220.

30. Willcocks SJ, Stabler RA, Atkins HS, Oyston PF, Wren BW. 2018. High-throughput analysis of Yersinia pseudotuberculosis gene essentiality in optimised in vitro conditions, and implications for the speciation of Yersinia pestis. BMC Microbiol 18:46.

31. Sun Y, Fukamachi T, Saito H, Kobayashi H. 2012. Adenosine deamination increases the survival under acidic conditions in Escherichia coli. J Appl Microbiol 112:775-81.

32. Nygaard P. 1978. Adenosine deaminase from Escherichia coli. Methods Enzymol 51:508-12.

33. Mutalik VK, Novichkov PS, Price MN, Owens TK, Callaghan M, Carim S, Deutschbauer AM, Arkin AP. 2019. Dual-barcoded shotgun expression library sequencing for high-throughput characterization of functional traits in bacteria. Nat Commun 10:308.

34. Baba T, Ara T, Hasegawa M, Takai Y, Okumura Y, Baba M, Datsenko KA, Tomita M, Wanner BL, Mori H. 2006. Construction of Escherichia coli K-12 in-frame, single-gene knockout mutants: the Keio collection. Mol Syst Biol 2:2006 0008. 
35. Kitagawa M, Ara T, Arifuzzaman M, loka-Nakamichi T, Inamoto E, Toyonaga H, Mori H. 2005. Complete set of ORF clones of Escherichia coli ASKA library (a complete set of E. coli K-12 ORF archive): unique resources for biological research. DNA Res 12:291-9.

36. Rodionova IA, Goodacre N, Babu M, Emili A, Uetz P, Saier MH, Jr. 2018. The Nitrogen Regulatory PII Protein (GInB) and N-Acetylglucosamine 6-Phosphate Epimerase (NanE) Allosterically Activate Glucosamine 6-Phosphate Deaminase (NagB) in Escherichia coli. J Bacteriol 200.

37. Rodionova IA, Li X, Plymale AE, Motamedchaboki K, Konopka AE, Romine MF, Fredrickson JK, Osterman AL, Rodionov DA. 2015. Genomic distribution of B-vitamin auxotrophy and uptake transporters in environmental bacteria from the Chloroflexi phylum. Environ Microbiol Rep 7:204-10.

38. Overbeek R, Olson R, Pusch GD, Olsen GJ, Davis JJ, Disz T, Edwards RA, Gerdes S, Parrello B, Shukla M, Vonstein V, Wattam AR, Xia F, Stevens R. 2014. The SEED and the Rapid Annotation of microbial genomes using Subsystems Technology (RAST). Nucleic Acids Res 42:D206-14.

39. Crooks GE, Hon G, Chandonia JM, Brenner SE. 2004. WebLogo: a sequence logo generator. Genome Res 14:1188-90.

40. Cho BK, Knight EM, Palsson BO. 2006. PCR-based tandem epitope tagging system for Escherichia coli genome engineering. Biotechniques 40:67-72.

41. Latif H, Federowicz S, Ebrahim A, Tarasova J, Szubin R, Utrilla J, Zengler K, Palsson BO. 2018. ChIPexo interrogation of Crp, DNA, and RNAP holoenzyme interactions. PLoS One 13:e0197272.

42. Rodionova IA, Goodacre N, Do J, Hosseinnia A, Babu M, Uetz P, Saier MH, Jr. 2018. The uridylyltransferase GInD and tRNA modification GTPase MnmE allosterically control Escherichia coli folylpoly-gamma-glutamate synthase FolC. J Biol Chem 293:15725-15732.

\section{Figures}




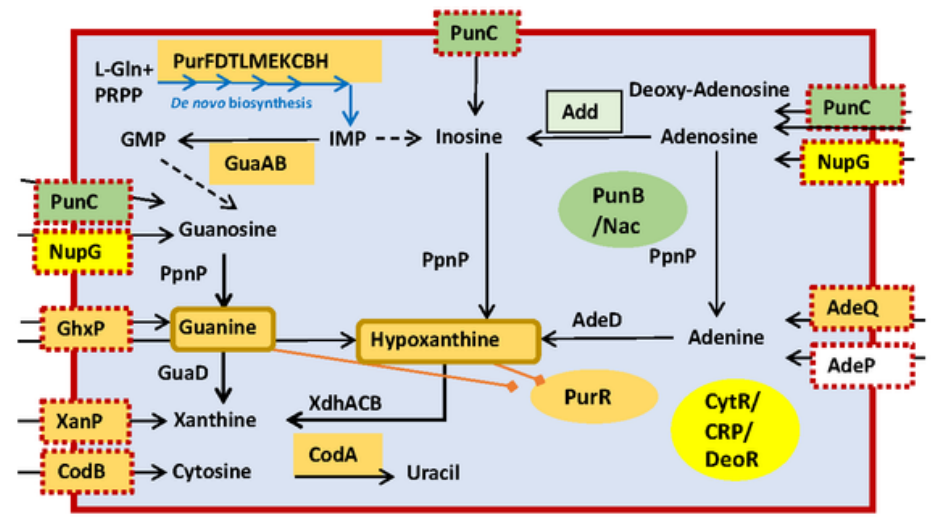

\section{Figure 1}

Metabolic pathways and transcriptional regulons for purine biosynthesis and purine/pyrimidine uptake and salvage genes in E. coli. The de novo purine biosynthesis pathway is shown by blue arrows. Other enzymes involved the purine and pyrimidine metabolism are shown by black arrows. Nucleoside uptake transporters are shown have dashed boxes. PurR is a transcriptional repressor of most of the purine biosynthesis and salvage genes in response to hypoxanthine and guanine. The PurR-regulated genes are highlighted in orange. The purine uptake transporter NupG is under transcriptional control of CytR, DeoR and CRP (shown in yellow). The novel purine transporter PunC (YdhC), described in this study, is controlled by the LysR-family activator PunR ( $\mathrm{YdhB}$ ) and Nac, as highlighted in green. Abbreviations: IMP, inosine monophosphate; AMP, adenosine monophosphate; GMP, guanosine monophosphate. 


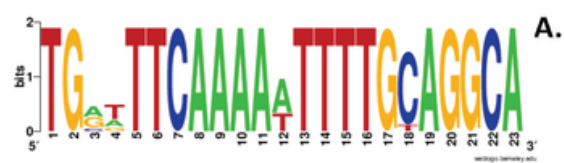

Fig.2

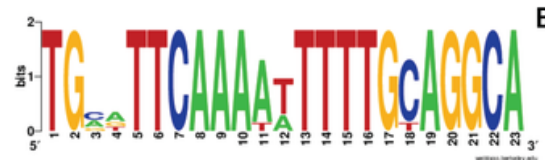

B.

F.
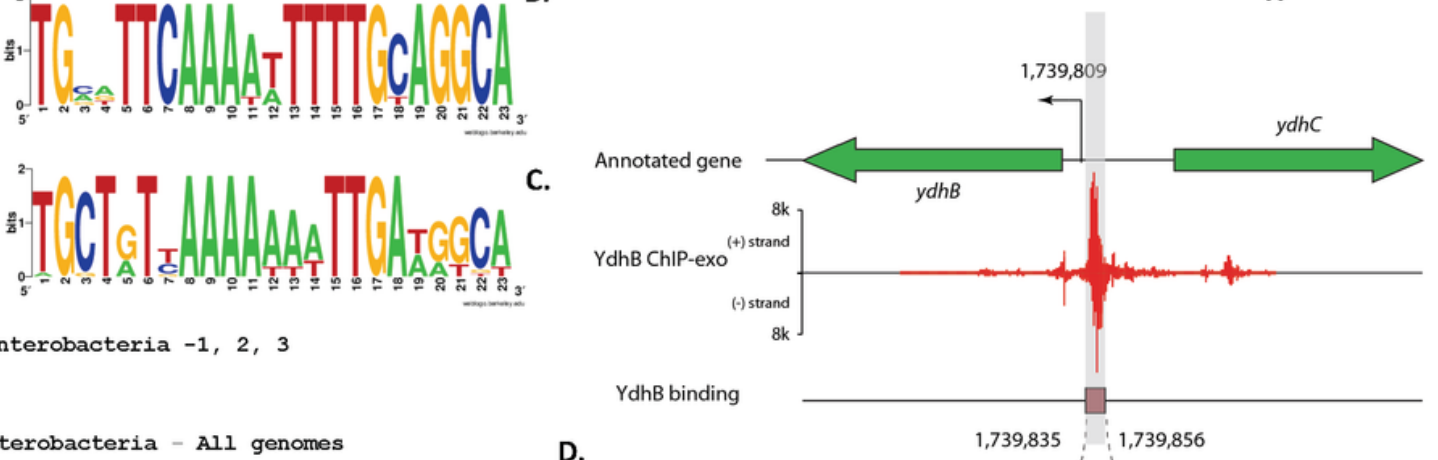

Enterobacteria - All genomes

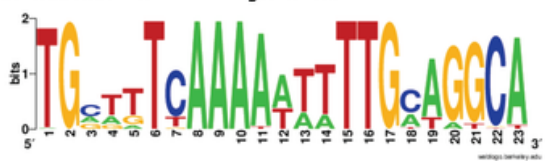

D.

$1,739,835: 1,739,856$

E.

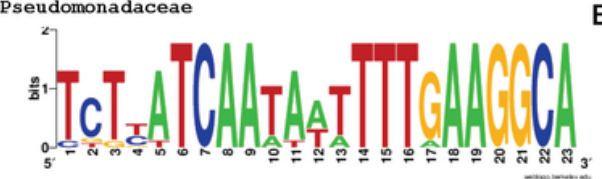

\section{Figure 2}

The predicted PunR binding site motifs in various groups of Enterobacteria and Pseudomonas spp. as identified by a phylogenetic footprinting approach (A-E). (F) Identification of the PunR binding region using ChIP-exo. Sequence logos were generated based on the multiple alignments of PunR binding sites from respective groups of Enterobacteria or Pseudomonas genomes (see Figure S2) using the WebLogo tool. The representative groups for Enterobacteria -1, 2, 3 and Pseudomonadaceae are described in Fig. S2. 


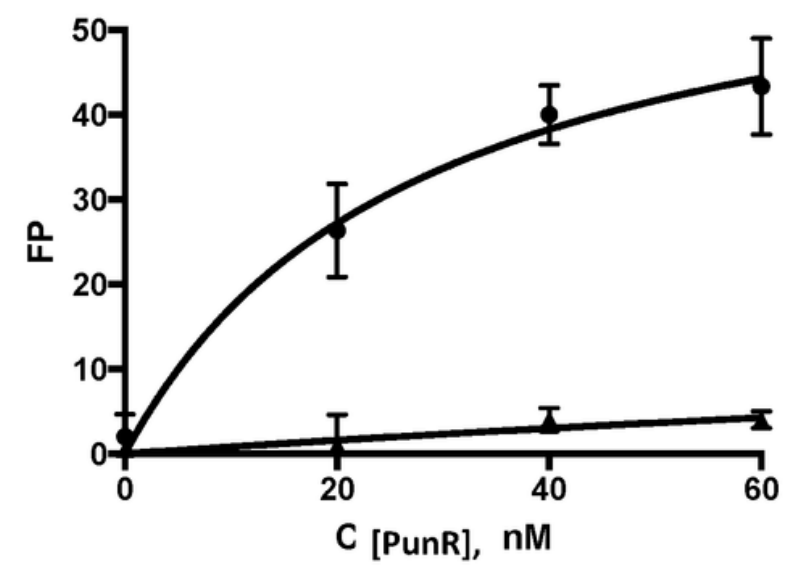

Fig.3

\section{Figure 3}

DNA binding assays with PunR. Binding of purified PunR protein to the predicted DNA sequence upstream of punC was assessed by the fluorescent polarization assay in the presence of $0.35 \mathrm{mM}$ adenine. 
A.

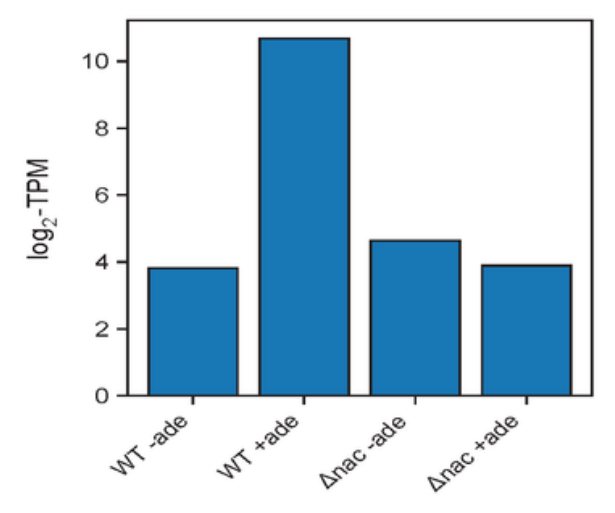

Fig.4
B.

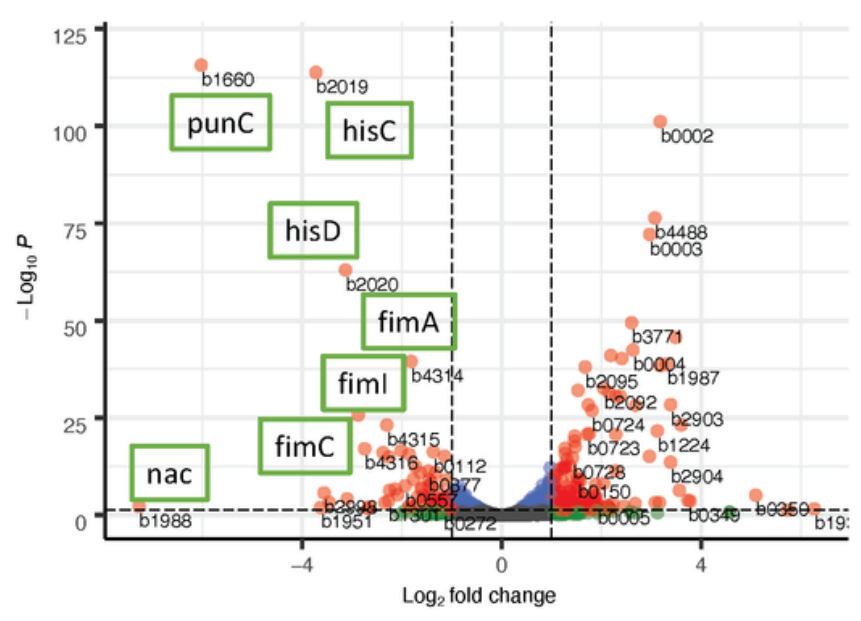

Total $=4355$ variables

\section{Figure 4}

RNA-Seq analysis for the punC mRNA expression. (A) E. coli MG1655 and nac mutant grown in M9 medium in the absence or presence of $10 \mathrm{mM}$ adenine. (B) mRNA differential expression for nac mutant and wild type in E. coli MG1655. Volcano plot comparing the log-fold expression change of genes against the adjusted p-value for the nac mutant and WT MG1655 grown at M9 supplemented with $10 \mathrm{mM}$ adenine. Genes in red dots are differentially expressed. punC (b1660) is the gene with the strongest expression change in addition to hisG (b2019) and hisD (b2020) histidinol dehydrogenase (L-histidine biosynthesis), fimA (b4314), fiml (b4315), fimC (b4316). 
Fig.5

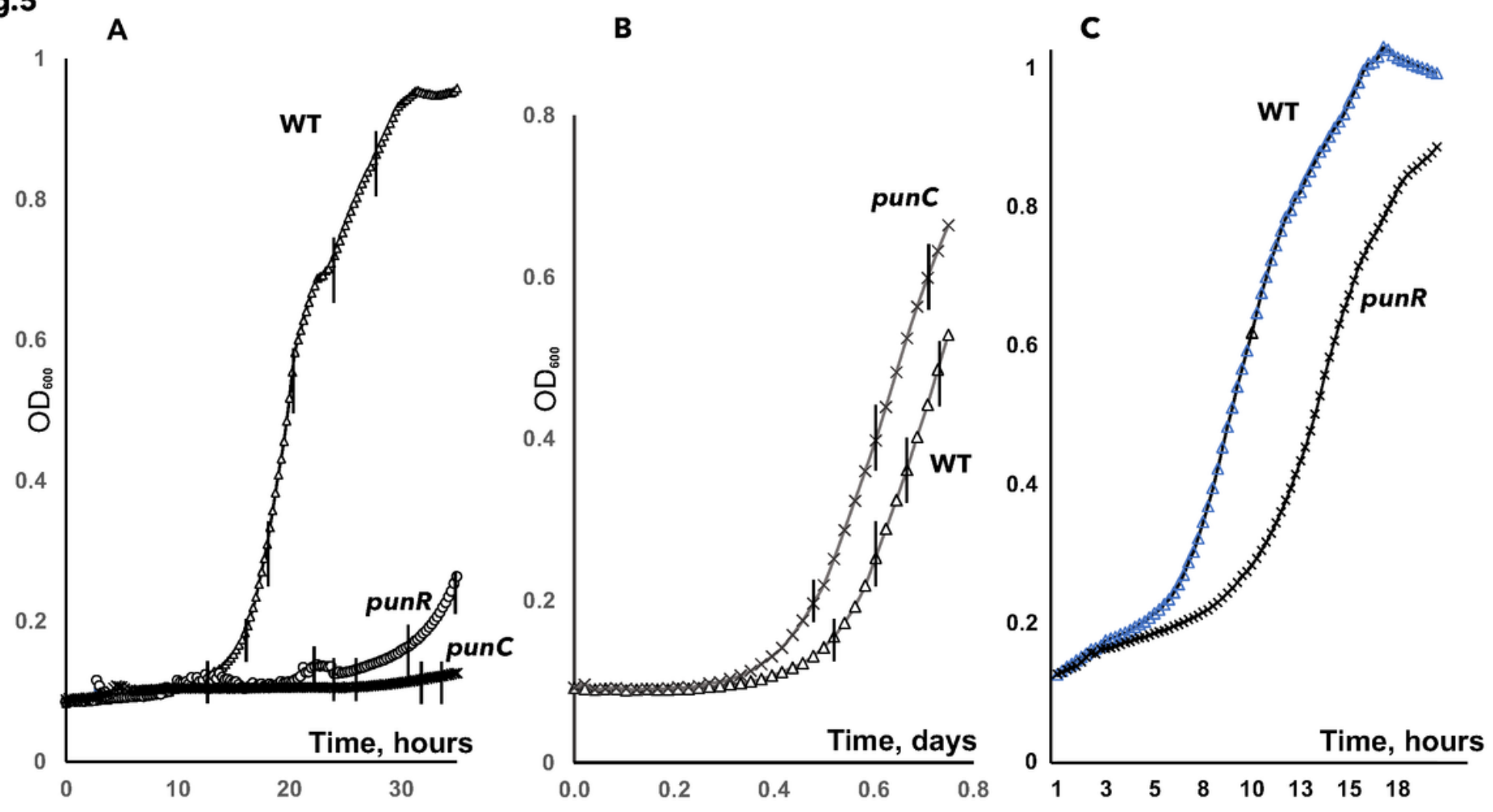

Figure 5

Growth phenotypes of the punR and punC knockout strains in $\mathrm{M} 9$ medium with $0.2 \%$ glucose as sole carbon source and (A) $2.5 \mathrm{mM}$ adenosine as the sole nitrogen source (B) $\mathrm{NH} 4 \mathrm{Cl}$ as the nitrogen source. The growth of the punR $(0)$ and punC $(X)$ deletion strains compared to the wild type E. coli BW25113 $(\Delta)$ strain (C) Growth of E. coli MG1655 wild type $(\Delta)$ and punR $(0)$ deletion strain in the same medium as in A. 
Fig.6

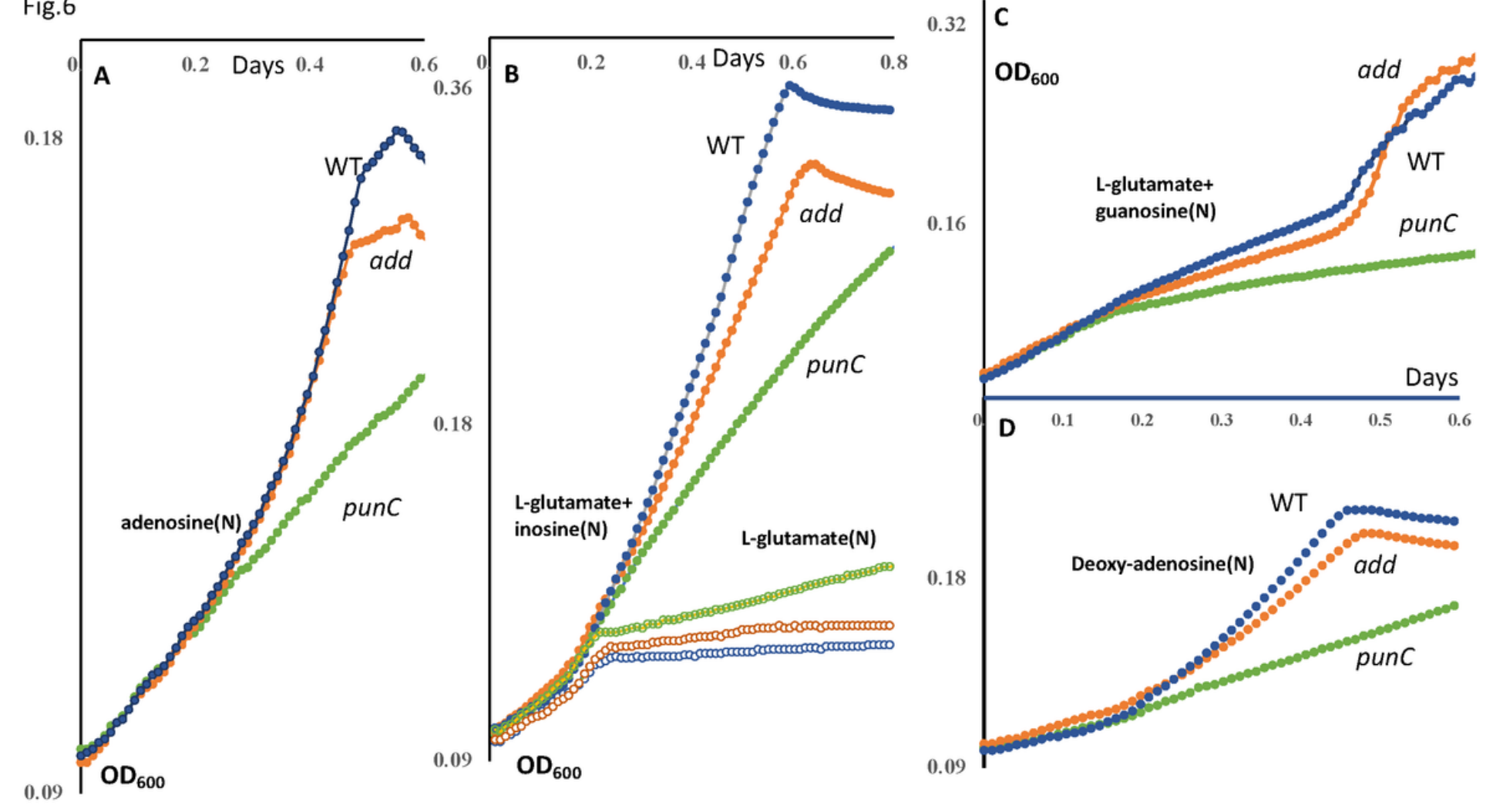

Figure 6

Growth phenotypes of the add and punC knockout strains in M9 medium with $0.4 \%$ glycerol as the carbon source and various nitrogen sources as follows: (A) $2.5 \mathrm{mM}$ adenosine as sole nitrogen source (B) $5 \mathrm{mM}$ L-glutamate and inosine, or $5 \mathrm{mM} \mathrm{L-glutamate} \mathrm{(empty} \mathrm{circles} \mathrm{with} \mathrm{strains} \mathrm{indicated} \mathrm{with}$ corresponding color) (C) $5 \mathrm{mM} \mathrm{L-glutamate} \mathrm{and} \mathrm{guanosine} \mathrm{(D)} 5 \mathrm{mM}$ 2-deoxyadenosine as sole nitrogen source. The growth of the punC (green circles) and add (orange line, circles) deletion strains compared to the wild type E. coli BW25113 (dark blue line, circles) strain.

\section{Image not available with this version}

\section{Figure 7}

Growth phenotypes of the E. coli BW25113 and punC knockout strains in the Biolog plate PM12B supplemented with $1 \mathrm{mM}$ inosine, the resistance to $(A, B)$ sulfathiazole_8X and $1 X$ and $(D, C)$ sulfamethoxazole_8X and $1 \mathrm{X}$ (folate antagonist).

\section{Supplementary Files}


This is a list of supplementary files associated with this preprint. Click to download.

- FigS2PunC.pdf

- FigS1PunC.pdf

- Figs3PunC.pdf 